\title{
Experimental determination of fatigue life of automotive jounce bumper
}

\begin{abstract}
It is evident that most rubber components in the automotive industry are subjected to repetitive loading. Vigorous research is needed towards improving the safety and reliability of the components. The study is conducted on an automotive rubber jounce bumper with a rubber hardness of 60 IRHD. The test is conducted in displacement controlled environment under compressive load. The existing models by Kim, Harbour, Woo and Li are adopted to predict the fatigue life. The experimental results show strong similarities with the predicted models.
\end{abstract}

Keyword: Rubber; Life prediction; Repeated loading; Jounce bumper; Fatigue failure 